\title{
Projets cliniques territoriaux des CSSS et participation des acteurs de la société civile
}

\author{
Alex Ellyson \\ Université de Sherbrooke \\ Denis Bourque \\ Professeur \\ Université du Québec à Chicoutimi
}

\section{Introduction}

Cet article est issu d'une recherche ${ }^{1}$ exploratoire portant sur l'implication de CDC (Corporation de développement communautaire) du Québec dans des processus d'élaboration de projets cliniques de CSSS (Centre de santé et de services sociaux). Trente responsables de CDC (sur un total de 43 au Québec) ont été interviewées à l'automne 2006. Il en ressort que 17 étaient impliquées dans un processus de projet clinique avec un CSSS et que 13 ne l'étaient pas. Nous désirions explorer: 1) les formes de participation des CDC et des autres acteurs dans les projets cliniques; 2) les impacts appréhendés des projets cliniques sur les organismes communautaires, sur les déterminants sociaux de la santé, ainsi que sur la pratique des organisateurs communautaires des CSSS. Sur le plan de la méthodologie, nous avons eu recours à des

\section{Les projets cliniques}

La loi 25 adoptée en décembre $2003^{2}$ a conduit à la création de 95 Centres de santé et de services sociaux (CSSS) qui sont généralement le résultat d'une fusion entre CLSC, CHSLD (Centre d'hébergement et de soins de longue durée) et CHSCS (Centre hospitalier de soins généraux et spécialisés) entrevues semi-dirigées auprès de 30 dirigeantes de CDC du Québec. L'analyse de contenu s'est fait selon un mode de type catégoriel à partir d'un résumé écrit des entrevues. Nous avons également procédé à l'analyse documentaire de divers textes relatifs aux projets cliniques. Cet article présente les résultats de cette recherche et analyse les principaux enjeux que soulèvent les projets cliniques pour le milieu communautaire concerné. Notons que cette recherche a des limites, car elle ne comporte pas un échantillon scientifiquement représentatif des CDC du Québec, ni des projets cliniques élaborés par les 95 CSSS. Il convient aussi de préciser que nous avons limité notre analyse à la période d'élaboration des projets cliniques et que, par conséquent, nous n'avons pas étudié leur contenu, ni leur mise en œuvre.

d'un territoire donné. Les CSSS ont le mandat de mettre sur pied et coordonner un réseau local de services. Pour ce faire, le CSSS est responsable de définir un projet organisationnel et clinique visant à répondre aux besoins de santé et de bien-être de la population du territoire à partir de divers 
services adaptés aux réalités locales, articulés entre eux et qui englobent l'ensemble des interventions dont la promotion-prévention, le diagnostic, le traitement, le suivi, l'adaptation et le soutien à l'intégration sociale, la réadaptation et le soutien en fin de vie $^{3}$. L'article 99,5 de la loi $83^{4}$ donne le mandat suivant aux CSSS :

Aux fins de définir son projet clinique et organisationnel, une instance locale [CSSS] doit, pour le territoire de son réseau local, mobiliser les établissements offrant des services spécialisés et sur spécialisés, les divers groupes de professionnels, les organismes communautaires, les entreprises d'économie sociale, les ressources privées et les intervenants des autres secteurs d'activité ayant un impact sur les services de santé et les services sociaux et s'assurer de leur participation.

Pour chacun des neuf programmes services ${ }^{5}$, le projet clinique consiste à :

1- Établir le portrait des besoins de santé et de bien-être de la population du territoire;

2- Inventorier les ressources et services actuellement disponibles pour répondre à ces besoins;

3- Analyser les écarts à combler pour atteindre les objectifs d'accès, de continuité et de qualité;

4- Identifier les modèles cliniques et les collaborations fructueuses déjà implantés qu'il faut maintenir et renforcer;
5- Choisir, s'il y a lieu, d'autres modèles à intégrer pour combler les écarts dans l'atteinte des objectifs retenus;

6- Préciser les éléments de l'offre de service et les paramètres devant être contenus dans les ententes de services, ainsi que préciser les rôles et les responsabilités des différents acteurs $^{3}$.

Les modalités de collaboration entre le réseau public et les organismes communautaires passeront, entre autres, par des ententes de services. À ce chapitre, le MSSS (Ministère de la Santé et des Services sociaux) stipule que les organismes communautaires ont, sur une base libre et volontaire, à définir avec leur CSSS les modalités de leur collaboration pour assurer une offre de services intégrés à la population de leur territoire dans le respect de l'autonomie de ces organismes ${ }^{3}$. Ces ententes de services peuvent cependant impliquer le versement de subventions et une reddition de comptes pour une prestation de services prédéfinis dans le cadre de réseaux intégrés ou continuums de services. Les ententes de services passent ainsi du statut de pratique marginale et peu normée à celui d'élément central et standardisé des nouveaux rapports entre CSSS et organismes communautaires (Bourque, 2007). Ces nouveaux rapports, issus de la mise en place des CSSS, appellent des relations plus hiérarchiques que les relations qui avaient jusque-là généralement cours entre les établissements publics de première ligne et les organismes communautaires.

\section{Trois positions en présence}

Parmi les chercheurs et les acteurs du mouvement communautaire, on note trois grandes tendances relativement aux projets cliniques. Une première tendance que l'on pourrait qualifier d'opposition se caractérise par des objections de fonds à la création des réseaux locaux de services, aux projets cliniques ainsi qu'aux ententes de services. Les réserves en regard des ententes de services ne sont pas nouvelles, mais 
Parmi les chercheurs et les acteurs du mouvement communautaire, on note trois grandes tendances relativement aux projets cliniques.

l'opposition aux réseaux locaux de services et à toute forme de collaboration formelle entre le communautaire et les CSSS est plus récente $^{6}$. La Coalition des TROC (Table régionale des organismes communautaires) remet ainsi en question la participation du communautaire à toute forme d'entente de collaboration, même celle qui n'implique pas de financement, car «elles font appel aux services de l'organisme communautaire dans le cadre de sa mission, mais sans y attacher de financement supplémentaire... [et font en sorte] ...d'assujettir une partie du budget du PSOC [programme de soutien aux organismes autonomes] de l'organisme à des services de responsabilité gouvernementale $»^{6}$.

Même la conclusion volontaire de telles ententes de collaboration ou de services, ou la participation aux continuums de services, est à rejeter, car ils signifient l'intégration à l'offre de services publics. Dans le cas contraire, l'organisme communautaire signataire de telles ententes devrait réclamer les mêmes conditions de travail que celles en vigueur dans le CSSS. Selon les tenants de cette position, le partenariat entre les organismes communautaires et le CSSS est perçu comme une menace potentielle pouvant mener à l'assujettissement du social au médical de même qu'à la perte de la légitimité des organismes communautaires qui pourraient de retrouver cantonnés au rôle de sous-traitants de l'administration publique.

Une seconde tendance serait la tendance complémentariste ${ }^{7}$ ou affairiste qui répond à une demande et à des pratiques de la part d'une partie du mouvement communautaire et des entreprises d'économie sociale pour renforcer leur intégration aux politiques et programmes publics souvent pour des fins de survie financière. Cette tendance est bien représentée par un courant des entreprises d'économie sociale en aide-domestique dont les rapports avec les établissements publics au palier local les situent dans une relation de quasi-sous-traitance, particulièrement au niveau des activités d'aide à la personne, incluant les bains et les soins d'hygiène ${ }^{8}$. La défense des intérêts organisationnels constitue le moteur des rapports d'affaires en présence. Cette position appréhende donc le partenariat entre le secteur public et le mouvement communautaire essentiellement selon une perspective managériale, c'est-àdire en termes de contractualisation d'ententes de services pour la prestation de services. Caillouette et coll. ${ }^{9}$, y réfère en termes de paradigme de régulation marchande (la construction des rapports mise explicitement sur les intérêts particuliers des acteurs, et ce, par le biais de mécanismes de marché). L'État et ses institutions, sans devoir de dialogue avec le mouvement communautaire, prennent alors les décisions dont l'identification des fournisseurs de services, la détermination des modalités de prestation de ces services, ainsi que les normes de contractualisation à mettre en place.

Une troisième tendance en présence serait la tendance stratégique présente chez certains chercheurs et parmi une partie du mouvement communautaire. Celle-ci consiste à prendre acte de manière critique des courants de territorialisation et de partenariat institutionnalisé des services d'intérêt général (dont ceux en santé et services sociaux) non pas comme une mode passagère, mais comme un changement de paradigme et une caractéristique du nouveau mode de développement ${ }^{10}$. À ce titre, comme le souligne Lévesque, la question est moins de savoir s'il faut être pour ou contre ces changements, «que de s'interroger sur les 
conditions nécessaires pour que ces nouvelles formes prolongent et même approfondissent la recherche de l'intérêt général et de la solidarité $»^{10}$. Cette tendance se concentre donc sur les modalités entourant la mise en place des réseaux locaux de services, des projets cliniques et des ententes de services afin qu'ils se réalisent dans les conditions les plus favorables aux valeurs portées par les organismes communautaires ainsi qu'aux besoins et aspirations des milieux et des populations concernés. Contrairement à la tendance «affairiste», la tendance «stratégique » compose à la fois avec les intérêts organisationnels en cause, mais aussi avec une représentation de l'intérêt général qui ne passe pas uniquement par des arrangements centraux au niveau de l'État et des politiques publiques, mais aussi par des arrangements entre les acteurs locaux prenant en compte les particularités locales et territoriales. Caillouette et coll. ${ }^{9}$ parlent alors de paradigme de régulation partenariale (les acteurs de la société civile sont inclus dans le développement de leur territoire) et d'un espace local important à investir par la société civile, puisque reposant sur la redéfinition (au besoin conflictuel) des rapports entre les acteurs permettant une communauté plurielle d'actions.

\section{Analyse des 17 CDC participantes au(x) projet(s) clinique(s)}

Cette partie concerne les 17 entrevues menées auprès d'autant de responsables de CDC afin d'explorer l'implication de ces organismes dans des démarches de projets cliniques avec le CSSS. L'implication de la presque totalité de ces CDC remonte au début des démarches entreprises par chacun des 17 CSSS. Dans la majorité des cas, cette participation découle de l'initiative du CSSS qui a convié les CDC à se joindre au processus d'élaboration des projets clinique sous différents motifs. Dans certains cas, les CSSS voulaient connaître l'opinion du milieu communautaire relativement à une proposition de plan d'élaboration des projets cliniques. Dans d'autres situations, les CDC étaient invitées à participer à des rencontres consultatives ou exploratoires, pour identifier la manière dont allait être abordée la question des projets cliniques. Les processus les plus participatifs sont ceux qui ont été décrits comme mettant dès le départ à contribution des organismes communautaires dans la détermination du cheminement et des règles du jeu devant mener à l'élaboration du projet clinique.

Les CDC ont généralement joint des groupes de travail prenant différentes formes, selon les CSSS : comités de travail, tables de concertation, forums, etc. Ces groupes de travail étaient principalement centrés sur l'amélioration des services offerts par le CSSS et par les autres dispensateurs de services de son territoire, ainsi que par des clientèles et des problématiques bien spécifiques. D'une région à l'autre, ces groupes de travail se sont donc vus confier de multiples mandats, de l'élaboration des modalités entourant l'implantation des réseaux locaux de services à l'analyse de l'offre de services, en passant par l'identification de clientèles cibles, les bilans, les recommandations, la planification, la priorisation, etc. Il en va de même pour les différents projets cliniques qui, dépendamment les régions, prennent la couleur de programmes services ou de chantiers, chacun concerné par des préoccupations aussi variées que la délinquance, la perte d'autonomie, le logement social, la sécurité alimentaire, l'intégration des communautés ethnoculturelles, etc.

En termes de structure, on peut toutefois résumer le mode de fonctionnement le plus couramment utilisé à comité de «pilotage » et 
un comité d' « orientation ». Le premier (comité de pilotage) est principalement composé de cadres et de membres du conseil d'administration des CSSS. Il fait souvent figure d'instance décisionnelle. Le second (comité d'orientation) regroupe différents acteurs de la communauté. Il a typiquement pour mandat de chapeauter diverses tables de concertation qui elles, sont constituées en fonction de thèmes bien particuliers. Dans l'ensemble des processus d'élaboration des projets cliniques, les CDC ont souvent joué un rôle d'interface entre les organismes communautaires autonomes et les acteurs institutionnels.

Les dirigeantes de CDC interviewées estiment que les démarches d'implantation des projets cliniques comportent des impacts (actuels ou prévisibles) tout autant positifs que négatifs pour les organismes communautaires. Sur le plan des avantages, on retient que les démarches d'implantation des projets cliniques sont souvent perçues comme un lieu de rencontre pertinent entre les acteurs du secteur communautaire et ceux du secteur public, qui se voient offrir l'opportunité de se sensibiliser mutuellement à leurs préoccupations respectives. Notons que le renforcement de la concertation et du partenariat entre le CSSS et les organismes communautaires de son territoire est un scénario qui semble se concrétiser dans certaines régions autour de l'identification de priorités locales et de projets communs.

Concernant les impacts de type négatif, on remarque que les thèmes ressortis sont majoritairement liés à la question du financement et de l'autonomie des organismes communautaires. Les responsables de CDC redoutent notamment de voir certains organismes communautaires modifier leur mission en fonction des ententes de services. Plusieurs appréhendent également l'éventuelle prégnance de la reddition de compte, en même temps que de voir leurs membres se transformer en main-d'œuvre à bon marché. Une des interrogations centrales est de savoir si les CSSS auront recours à des mécanismes de suivi de gestion leur permettant d'assumer leurs responsabilités, tout en respectant la réalité et le mode d'organisation des services communautaires.

Par ailleurs, la plupart des responsables de CDC interviewées estiment que la principale force de la démarche d'élaboration des projets cliniques est reliée à la question de la concertation. Dans l'ensemble, elles sentent que le point de vue du communautaire a été à la fois entendu, et respecté. La présence d'un climat d'ouverture, l'intensité des échanges et la circulation de l'information ont été mentionnées pour illustrer leur propos. Elles ont apprécié que les démarches d'élaboration de projets cliniques conduisent à dresser un portrait sociosanitaire global de leur territoire, et à déterminer des priorités locales.
Plusieurs estiment que le processus d'élaboration des projets cliniques a été quelque peu précipité, et que cela a eu des répercussions néfastes sur différentes dimensions de la consultation, dont sa qualité, sa pertinence, et la participation.
En ce qui concerne les désavantages, une insatisfaction souvent mentionnée concerne la vitesse à laquelle ces démarches ont été accomplies. Plusieurs estiment que le processus d'élaboration des projets cliniques a été quelque peu précipité, et que cela a eu des répercussions néfastes sur différentes dimensions de la consultation, dont sa qualité, sa pertinence, et la participation. Une autre insatisfaction souvent exprimée se rapporte au coût de la démarche qui représente un investissement de temps et d'argent considérable. Certaines responsables de CDC s'interrogent également sur le bien-fondé de la 
démarche d'élaboration des projets cliniques, entre autres en termes de résultats. Des intervenantes questionnent aussi la validité et la pertinence des consultations qui en résultent. Elles ont également identifié la lourdeur des structures et des modes de fonctionnement public en tant que frein à l'action, et à la consultation.

Le tableau suivant reprend les principaux énoncés concernant les processus d'élaboration des projets cliniques du point de vue des répondantes des CDC.

Tableau \# 1 : Synthèse des énoncés quant au processus d'élaboration des projets cliniques

\begin{tabular}{|c|c|}
\hline \multicolumn{2}{|c|}{ Les impacts } \\
\hline $\begin{array}{l}\text { Type avantage: } \\
\text { - Lieu de rencontre et réseautage } \\
\text { - Occasion de sensibilisation mutuelle } \\
\text { - Concertation sur des priorités locales et des } \\
\text { projets communs }\end{array}$ & $\begin{array}{l}\text { Type désavantage : } \\
\text { - Énergivore en temps et en argent } \\
\text { - Risqué pour l'autonomie du } \\
\text { communautaire avec les ententes de } \\
\text { services et la reddition de compte (sous- } \\
\text { traitance) } \\
\text { - Faiblesse des moyens (argent neuf) }\end{array}$ \\
\hline \multicolumn{2}{|c|}{$\begin{array}{l}\text { Forces du processus : } \\
\text { - Participation large et qualité de la concertation qui joue un rôle rassembleur } \\
\text { - Climat d'ouverture, d'échange, d'écoute et de respect du point de vue du communautaire } \\
\text { - Identification du portrait sociosanitaire global du territoire et des priorités locales }\end{array}$} \\
\hline \multicolumn{2}{|c|}{$\begin{array}{l}\text { Limites du processus : } \\
\text { - Vitesse et échéancier trop rapides qui menacent qualité, pertinence et participation } \\
\text { - Incertitude quant aux résultats concrets } \\
\text { - Disproportion entre le poids des priorités locales VS les priorités ministérielles } \\
\text { - Lourdeur des structures et modes de fonctionnement des CSSS (surtout ceux récemment fusionnés) } \\
\text { - Tendance administrative à l'uniformisation des territoires des CSSS issus de fusions }\end{array}$} \\
\hline
\end{tabular}

\section{La contribution des organisateurs communautaires et place de la prévention et des déterminants sociaux de la santé dans les projets cliniques}

La contribution des organisateurs communautaires (o.c.) dans les démarches d'élaboration des projets cliniques varie grandement d'une région à l'autre. Ils sont impliqués dans la plupart des projets, mais pour accomplir des mandats différents. Selon les CSSS, on observe que les o.c. occupent des rôles aussi variés que la coordination ou l'animation, en passant par la mobilisation, le soutien technique, la recherche, etc. Précisons que généralement, ce sont des membres du personnel-cadre du CSSS (direction générale et direction de programme) qui dirigent les travaux liés à l'élaboration des projets cliniques. Concernant la prévention et les déterminants sociaux de la santé dans les projets cliniques, on retient deux tendances : leur présence via les organismes communautaires, ou leur absence en raison de la prédominance du médico-curatif. On note également que la prévention et les déterminants sociaux de la santé sont des enjeux importants des projets cliniques, mais que le contexte organisationnel est un frein majeur (priorités ministérielles, approche par programme, ententes de gestion). 


\section{Discussion}

\section{On peut dégager trois tendances chez les CSSS quant à l'élaboration des projets cliniques.}

Les CSSS sont maintenant non seulement imputables des services qu'ils offrent, mais aussi de la coordination de ceux offerts par leurs partenaires. Parallèlement, ils doivent favoriser la participation des ressources du milieu dans l'élaboration et la mise en œuvre des projets cliniques MSSS $^{11}$. On peut dégager trois tendances chez les CSSS quant à l'élaboration des projets cliniques. Une première qualifiée d'attentiste, car basée sur une absence d'action ou une action minimale (en 2006) qui se manifeste par une absence de sollicitation envers leur CDC ou le rejet d'une offre de participation de sa part. Une seconde D'une part, il faut considérer les profondes transformations associées aux fusions d'établissement qui touchent la majorité des CSSS et qui font que nombre d'entre eux sont toujours en pleine transition et réorganisation. Par ailleurs, les projets cliniques doivent s'inscrire dans les orientations bien spécifiques du MSSS et des Agences régionales, ce qui signifie la présence de priorités et de cibles prédéterminées (particulièrement en santé) qui s'imposent aux acteurs locaux. Soulignons que les propos de plusieurs dirigeantes de CDC allaient en ce sens. Les CSSS doivent donc naviguer au cœur d'une dynamique complexe (Duperré, 1992) $^{12}$ composée d'une logique descendante qui correspond aux priorités et cibles gouvernementales inscrites dans les ententes de gestion, et d'une logique ascendante qui correspond à l'aspiration des acteurs locaux de pouvoir déterminer les priorités et les stratégies d'action appropriées. $\mathrm{Au}$ centre, se retrouve les CSSS en interface entre les logiques descendante et ascendante, et en situation de double imputabilité soit vers le bas et leur milieu et vers le haut et l'appareil tendance en serait une administrative et de relations publiques qui s'incarne dans des pratiques qui consistent à faire du projet clinique une politique ou une procédure institutionnelle qui fera l'objet de consultations formelles une fois élaborée à l'interne (ou même confiée à un consultant). Une troisième tendance dite participative se caractérise par un investissement du CSSS dans un processus de concertation plus ou moins participatif vu comme une opportunité de développement autour d'enjeux non seulement institutionnels, mais aussi locaux et communautaires, selon la culture partenariale déjà présente au CSSS et dans son milieu. Cette dernière tendance est particulièrement intéressante, mais semble rencontrer des obstacles.

de planification régionale et nationale. En effet, les CSSS sont à la fois parties des politiques et programmes descendants et à ce chapitre ils doivent tenir compte des priorités qui en découlent. Mais ils sont aussi des acteurs du développement de leur milieu et à ce titre ils doivent contribuer à l'émergence des priorités locales, en tenir compte dans leurs projets cliniques, et favoriser l'actualisation des particularités et des choix locaux dans la mise en place des réseaux locaux de services. Le défi des CSSS est de se situer de manière efficace à la jonction entre les logiques descendantes et ascendantes, en assumant un rôle de leadership démocratique (Bourque, 2006) ${ }^{13}$.

À ce sujet, des responsables de CDC ont affirmé s'être sentis incluses et prises en compte dans l'élaboration des projets cliniques. Elles ont par ailleurs formulé des réserves sur la pertinence de ces démarches, notamment sur le plan de la validité de la consultation ainsi que des résultats potentiels. Autrement dit, tous les CSSS ont-ils les 
moyens (Fournier, 2007) ${ }^{14}$, la capacité et la volonté de mener à bien ces projets, tout en incluant, en tant que partenaires, les acteurs communautaires? Ces questions se posent, car pour l'instant, de nombreux éléments demeurent en suspens.

Ainsi, la mise en place des réseaux locaux de services instaure un contexte appelant à la conclusion d'ententes de services entre les CSSS et les organismes communautaires. Ceux-ci pourraient alors se retrouver à jouer à la fois le rôle de partenaires et de sous-traitants de l'instance locale (RRASMQ, 2005) ${ }^{15}$. On pourrait alors appréhender un renforcement des instances publiques au détriment de la société civile (regroupant entre autres les organismes communautaires et les entreprises d'économie sociale). En effet, dans un scénario plus négatif, celle-ci pourrait perdre sa légitimité en se voyant notamment confinée au rôle de sous-traitant de l'administration publique. Comme le suggèrent d'ailleurs Caillouette et coll. ${ }^{9}$ «Le danger d'un monologue institutionnel et un tantinet autoritaire de la part des CSSS est une réalité à envisager ». Par contre, comme le projet clinique «interpelle tous les partenaires et suscite leur adhésion et leur mobilisation ${ }^{11}$, ces auteurs font valoir qu'il « est difficile d'envisager l'élaboration de ces projets dans le cadre d'une imposition des vues des CSSS. Ces derniers auront sur chacun de leur territoire à composer avec les acteurs communautaires et d'économie sociale ${ }^{11}$. La création et la mise en œuvre des réseaux locaux de services pourraient donc représenter un espace stratégique à investir par les acteurs de la société civile. Selon ce scénario, cela pourrait non seulement leur permettre de renforcer leur pouvoir d'action, mais aussi s'avérer être une occasion de développer, avec le réseau public, des pratiques de partenariat et de collaboration fondées sur une communauté plurielle d'actions ${ }^{9}$.
Quel scénario sera le plus prégnant ? Les projets cliniques s'inscrivant dans une tendance participative offrent l'opportunité aux acteurs publics et communautaires de se sensibiliser mutuellement à leurs préoccupations respectives, tout en apprenant à mieux collaborer. Considérant les propos de plusieurs dirigeantes de CDC, on peut d'ailleurs penser que certains projets cliniques soient un levier incitant les divers acteurs en présence à travailler en concertation, selon les besoins locaux, et en fonction de logiques partenariales respectueuses des différences. Soulignons cependant que ce rapprochement entre les deux milieux comporte un danger pour le communautaire, soit celui de voir sa légitimité et sa spécificité être «contaminées » par des approches institutionnelles ou technocratiques, propres aux établissements publics. Considérant certains des principes formulés par le $\mathrm{MSSS}^{3}$ pour l'élaboration des projets cliniques (standardisation des pratiques, hiérarchisation des services, ententes de services, etc.), et considérant la pression à laquelle sont soumis les CSSS (ententes de gestion, données probantes, etc.), il $y$ a effectivement risque d'instrumentalisation et/ou de standardisation des pratiques communautaires, ce qui dénaturerait évidemment la contribution originale $\mathrm{du}$ milieu communautaire (participation citoyenne, action sociale, vie associative démocratique, etc.).

Les propos des responsables de CDC démontrent par ailleurs que les personnes en place influencent la direction prise par les CSSS quant à la participation des organismes communautaires aux projets cliniques, et cela même si des lignes directrices sont émises par le MSSS. Globalement, le facteur humain constitue une variable non négligeable dans la dynamique de mise en place des réseaux locaux de services. 


\section{Conclusion}

Les projets cliniques et les réseaux locaux de services, comme l'ensemble de la réforme en santé et services sociaux, demeurent un processus en cours de réalisation. Nous avons examiné comment la phase d'élaboration d'un certain nombre de projets cliniques était perçue du point de vue d'un des acteurs communautaires impliqués. Il s'agit d'un angle de prise trop étroit pour pouvoir en tirer de conclusions d'ensemble sur les projets cliniques et les réseaux locaux de services. D'autres recherches sont requises pour documenter et analyser le contenu même des projets cliniques, leur phase de mise en œuvre, ainsi que les formes d'arrangements durables qu'ils instaurent entre les acteurs et leur impact sur les services et la santé des populations visées.

\section{Même si des tensions importantes ont toujours marqué les rapports entre le réseau public et les organismes communautaires, l'existence et le devenir de plusieurs d'entre eux sont liés à la pérennité du système public.}

Mais déjà, il ressort clairement que cette réforme comporte des enjeux majeurs pour les acteurs concernés et les services collectifs dans le domaine de la santé et des services sociaux. Pour les organismes communautaires, il en va de leur spécificité et de leur identité. Pour les CSSS, dans un contexte de forte remise en question du caractère public du système de santé et de services sociaux, une contreperformance, voire un échec, des réseaux locaux de services donneraient des munitions à ceux qui s'activent à privatiser le système sociosanitaire. Cet enjeu ne concerne pas seulement les établissements publics, mais aussi les organismes communautaires, car dans un scénario de privatisation et de marchandisation des services d'intérêt général on voit difficilement comment le communautaire pourrait en sortir gagnant. Même si des tensions importantes ont toujours marqué les rapports entre le réseau public et les organismes communautaires, l'existence et le devenir de plusieurs d'entre eux sont liés à la pérennité du système public. Dans ce sens, la réussite des réseaux locaux de services pourrait constituer un enjeu en elle-même pour les acteurs publics et communautaires concernés, en plus de l'enjeu qu'elle représente pour les populations visées en termes d'amélioration de leur condition sociosanitaire et des services d'intérêt général dans ce domaine. Or, la réussite des réseaux locaux de services et des projets cliniques qui s'y rattachent ne se prescrit ou ne se décrète pas d'autorité. Elle résulte d'un processus participatif et délibératif complexe qui n'est jamais épuisé dans une planification ou un projet clinique.

Les réseaux locaux de services s'inscrivent dans un mouvement plus large de territorialisation de la gestion et de la gouvernance des services d'intérêt général ${ }^{10}$ qui tire ses origines de deux sources : la transformation de la gestion publique au Québec; l'évolution des formes de réponse collective des communautés aux problèmes qui les confrontent. Les pratiques de territorialisation, dans lesquelles se situent les projets cliniques, se conjuguent sous deux registres qui se métissent sur le terrain: celui de l'instrumentalisation qui utilise le territoire et les communautés comme terreau pour l'implantation de priorités et programmes publics préconstruits; celui de la coconstruction à l'échelle du territoire d'une dynamique d'appropriation du développement où la contribution des politiques et pouvoirs publics est négociée et intégrée. Le défi est le réaliser sur une base territoriale une nouvelle gouvernance partagée ${ }^{10}$ basée sur une gestion démocratique du changement. Les CSSS du 
Québec pourraient jouer un rôle important dans la mise en place de cette nouvelle gouvernance partagée dans la mesure où ils ont une légitimité reconnue localement pour ce faire. Les projets cliniques et les réseaux locaux de services représenteront pour les CSSS un test déterminant à cet égard.

\section{Notes et références}

1 Le rapport de cette recherche, subventionnée par le Fonds québécois de recherche sur la société et la culture, sera disponible sous forme de cahier, en novembre 2007, sur le site internet de l'Alliance de recherche universitécommunauté (ARUC) : http://www.uqo.ca/aruc/

2 QUÉBEC (2003). Loi sur les agences de développement des réseaux locaux de services de santé et de services sociaux, Québec, 2003, chapitre 21.

3 MINISTÈRE DE LA SANTÉ ET DES SERVICES SOCIAUX: MSSS (2004a). Projet clinique : Cadre de référence pour les réseaux locaux de services de santé et de services sociaux, Document principal, Québec, Gouvernement du Québec, Octobre, 75 p, page 56.

4 QUÉBEC (2005). Projet de loi $n^{\circ} 83$, Loi modifiant la Loi sur les services de santé et les services sociaux et d'autres dispositions législatives, Québec, 2005.

5 Les neuf programmes-services sont : 1) Perte d'autonomie liée au vieillissement, 2) Services généraux, 3) Santé publique, 4) Santé mentale, 5) Jeunes en difficultés, 6) Santé physique, 7) Déficience physique, 8) Déficience intellectuelle et 9) Dépendances (alcool, toxicomanie, jeux).

6 GAGNON-LESSARD, L. et RIVARD. S. (2007). Analyse du document "Offre de service-Services psychosociaux généraux 2007-2012 »du MSSS, Coalition des TROC, Rencontre nationale du 13-14-15 février 2007. $10 \mathrm{p}$.

7 PROULX, J. (1997). Le partenariat entre l'État et les organismes communautaires dans le cadre de la Loi 120: l'enjeu de la complémentarité, Mémoire de maîtrise, Québec, Université Laval, 202 p.

8 FOURNIER, J. (2000). «Aide à domicile : pour que l'économie sociale demeure de l'économie sociale ", Nouvelles pratiques sociales, Vol. 13, no 2, 193-206.

9 CAILlOUETTE, J., GARON, S., ELLYSON, A. (2006). «Les enjeux de la redéfinition des rapports de l'État à la société civile dans la gouvernance du secteur de la santé et des services sociaux au Québec », dans FLEURY, M.-J., TREMBLAY, M., NGUYEN, H., BORDELEAU, L., Le système sociosanitaire au Québec : gouverne, régulation et participation, Montréal, Gaëtan Morin Éditeur, p. 451-465, 457.

10 LÉVESQUE, B. (2006). Une gouvernance partagée et un partenariat institutionnalisé pour la prise en charge des services d'intérêt général, Cahier C-13-2006, CRISES, UQAM, 26 p.

11 MINISTÈRE DE LA SANTÉ ET DES SERVICES SOCIAUX: MSSS (2004b). Projet clinique : Cadre de référence pour les réseaux locaux de services de santé et de services sociaux, Document résumé, Québec, Gouvernement du Québec, Octobre, 23 p.

12 DUPERRÉ, M. (1992). «Du discours à la réalité dans le partenariat public-communautaire en santé mentale: une expérience au Saguenay-Lac-Saint-Jean», Nouvelles Pratiques Sociales, vol. 5, n 2, Presses de l'Université du Québec, Québec, pp. 131-146.

13 BOURQUE, D. (2006). «Interfaces communautaire/réseau public : continuité et rupture», Économie et Solidarités, vol. 36, n², 13-26.

14 FOURNIER, Jacques (2007). Les C3S ont-ils les moyens de définir leur propre projet clinique?, http://rqiiac.qc.ca/fr/actualites_debats/chronique_jacques/article.asp?offset=10\&Id_articles=1039

15 RRASMQ : REGROUPEMENT DES RESSOURCES ALTERNATIVES EN SANTÉ MENTALE DU QUÉBEC (2005), Réseaux intégrés de services en santé mentale et enjeux des pratiques, Perron, N., 20 p. en ligne : http://www.rrasmq.com/Publications/Fevrier\%202006/Perron\%20NPS\%20RIS.pdf 